\title{
Trayectorias sexuadas, egresadas universitarias en educación virtual desde una mirada transversal
}

\section{Sexed virtual trajectories, university graduates in virtual education from a transversal perspective}

\author{
Patricia Graciela Sepulveda \\ https://orcid.org/0000-0003-1081-6401 \\ pgsepulveda@unq.edu.ar \\ Universidad Nacional de Quilmes | Argentina
}

\section{RESUMEN}

Diversos estudios, referidos a la población universitaria, mencionan el crecimiento de la matrícula femenina y al mismo tiempo su presencia en carreras que muestran relación con roles femeninos tradicionales. Carreras altamente feminizadas son la docencia, la enfermería, la obstetricia, asociadas con los roles de cuidado atribuidos a las mujeres, y resultan jerárquica y salarialmente infravaloradas.

Las trayectorias universitarias se consideran en tanto pertenecientes a personas sexuadas y teniendo en cuenta el impacto de los discursos, tecnologías y técnicas que forjan masculinidades y femineidades específicas en cada contexto social e histórico particular.

A partir de estas ideas y a la luz de la revisión de la bibliografía nos proponemos analizar información procedente de entrevistas en profundidad realizadas a egresados y egresadas de las distintas carreras de grado que ofrece la Universidad Nacional de Quilmes en su modalidad virtual.

Este artículo se centra en los relatos acerca de las trayectorias estudiantiles femeninas desarrolladas hasta convertirse en graduadas y muestran cómo aún en entornos virtuales las experiencias continúan siendo sexuadas y diferentes para varones y mujeres. Si bien se entiende que es un caso particular, se pretende aportar al conocimiento de las trayectorias de aquellos que logran convertirse en graduados y graduadas universitarias.

PALABRAS CLAVE trayectorias, virtualidad, relaciones de género, mujeres. 
KEY WORDS

trajectories,

virtual,

gender relationship,

women.

\section{ABSTRACT}

Various studies, referring to the university population, mention the growth of female enrollment and at the same time their presence in careers that show a relationship with traditional female roles. Highly feminized careers are teaching, nursing, midwifery, associated with the care roles attributed to women, and they are hierarchically and undervalued in salary.

University trajectories are considered as belonging to sexed persons and taking into account the impact of the discourses, technologies and techniques that forge specific masculinities and femininity in each particular social and historical context, and exclude by default any non-binary gender identity.

Based on these ideas and in light of the review of the bibliography, we propose to analyze information from in-depth interviews conducted with graduates of the different undergraduate degrees offered by the National University of Quilmes in its virtual modality.

This presentation focuses on the stories about female student trajectories developed to become graduates. Although it is understood that it is a particular case, it is intended to contribute to the knowledge of the trajectories of those who manage to become university graduates. 


\section{1- INTRODUCCIÓN}

En este artículo nos proponemos, a través del análisis de entrevistas a varones y mujeres estudiantes de carreras a distancia, aportar al conocimiento de las trayectorias de quienes logran graduarse. La incorporación de relatos en primera persona propone iluminar, a través de una reducción en la escala de observación, las subjetividades que animaron dichas trayectorias y cómo operaron las pedagogías de la sexualidad respecto de las masculinidades y feminidades esperadas, discutidas o asumidas por las y los estudiantes. Proponemos que aún en entornos virtuales de aprendizaje las experiencias sexuadas se hacen presentes ${ }^{2}$.

La perspectiva que aquí se asume incorpora los aportes de los estudios de género y la teoría feminista fundamentalmente referiremos a dos conceptos: conocimientos situados, objetividad encarnada (Haraway 1995) partimos de la idea que la investigación feminista no pretende desarrollar una objetividad que impone un tono neutro que es a la vez masculino, blanco, desencarnado y trascendente. Por el contrario señala la necesidad de reconocer la subjetividad de quien investiga, asumiendo que no hay conocimiento neutro. La objetividad feminista supone conocimientos situados. Lo que implica entender la producción de conocimiento como una situación atravesada por relaciones de poder y además localizada en contextos sociales, geográficos, políticos e históricos.

Nuestro enfoque asume, además, la importancia de las conceptualizaciones subjetivas, que son fruto de la experiencia de los y las agentes y de su reflexión sobre la construcción del mundo social. Esto implica estudiar el mundo, sin perder la perspectiva analítica del investigador, considerando dichas perspectivas; y no suscribir a enfoques que niegan las interpretaciones y la construcción personal e interpersonal de

$2 \quad$ Este artículo se maneja con categorías binarias, no porque asuma la inexistencia de otras identidades sexo-genéricas sino porque el sistema de registro universitario SIU solo incluía las categorías varón- mujer hasta abril de 2021. En esa fecha a través del trabajo articulado de varios organismos del Estado, se presentó una propuesta para incorporar la variable de identidad de géneros y diversidad en los diferentes módulos del Sistema de Información Universitaria (SIU) del Consejo Interuniversitario Nacional (CIN), en cumplimiento de la Ley $N^{\circ} 26.743$ de identidad de género. recuperado online de: https://www. cin.edu.ar/perspectiva-de-generos-y-diversidad-en-los-sistemas-siu/ 
significados, valores y hechos sociales que estos realizan. Apelar a los relatos de experiencia -creemos- permite acceder a los significados atribuidos.

Dejamos, en este trabajo, de lado posturas que entienden la sexualidad como biológica y el género como puramente construido, asumimos aquí que la diferencia sexual deviene de una construcción histórica y social y la institución escolar (en todos los niveles) contribuye a esta construcción tanto como a la de la sexualidad, entendida como heteronormativa y binaria, enclosetando a todas aquellas sexualidades disidente ${ }^{3}$.

\section{Dejamos, en este trabajo, de lado posturas que entienden la sexualidad como biológica y el género como puramente cons- truido, asumimos aquí que la diferencia sexual deviene de una construcción histórica y social y la institución escolar (en to- dos los niveles) contribuye a esta construcción tanto como a la de la sexualidad, entendida como heteronormativa y binaria, enclosetando a todas aquellas sexualidades disidente.}

Es la cultura, histórica, social y regionalmente situada, la que construye lo femenino y lo masculino y define los comportamientos esperables para cada uno, más allá de los rasgos anatómicos de las personas. Las representaciones colectivas así conformadas jerarquizan de manera desigual los comportamientos y expectativas a favor de lo masculino, relegando a las mujeres a un espacio de subordinación. A partir de ello, en nuestra indagación, nos preguntamos por el modo en que los testimonios analizados pusieron en evidencia o cuestionaron estas jerarquías y sí en espacios universitarios analizados se habilita la posibilidad de emergencia de sexualidades disidentes.

Este ensayo es solo un recorte y se inscribe en un proyecto de investigación que incluyó análisis estadísticos cuantitativos de la población constituida por todas las carreras, en modalidad virtual, respecto de estudiantes, performances, trayectorias y graduaciones que se extendió entre 2012 y 2019 en la Universidad Nacional de Quilmes. Si bien el recorte temporal de la investigación es previo a la situación de Pandemia, acercamientos posteriores a relatos estudiantiles dieron cuenta de la denominada "crisis de los cuidados" que castigaron con mucha más fuerza a las subjetividades feminizadas, lo que tuvo impacto en sus trayectorias y en la extensión temporal de estas, cuando no en

$3 \quad$ Eliana Vasquez y Claudia Lajud (2016) observaron la pervivencia en la escuela de la pedagogía del closet, pedagogía constituida a partir de un ideal de pureza binaria de los sexos que impone modelos hegemónicos y heterosexuales, con el consecuente enclosetamiento de todas aquellas manifestaciones de sexualidades disidentes (Vasquez y Lajud 2016, p 74) 
directos abandonos de asignaturas y de carreras. Sin embargo como esas trayectorias todavía están en estudio son referidas aquí sólo de modo tangencial.

Si bien el recorte temporal de la investigación es previo a la situación de Pandemia, acercamientos posteriores a relatos estudiantiles dieron cuenta de la denominada "crisis de los cuidados" que castigaron con mucha más fuerza a las subjetividades feminizadas, lo que tuvo impacto en sus trayectorias y en la extensión temporal de estas, cuando no en directos abandonos de asignaturas y de carreras. Sin embargo como esas trayectorias todavía están en estudio son referidas aquí sólo de modo tangencial.

\section{ALERTA, MUJERES EN LA EDUCACIÓN SUPERIOR}

La creciente feminización de la matrícula que se hizo evidente en la década de 1970, a fines de la década siguiente alcanzaba el 47\%. Puede afirmarse que una vez que las mujeres ingresaron a la Educación Superior (ES) su número creció más aceleradamente que el de los varones, sin embargo, el número de egresadas y estudiantes no se tradujo, de modo inmediato, en una mayor participación en las cátedras, en las políticas de las carreras, en un mayor desarrollo profesional o en la investigación.

Diversos estudios, referidos tanto a Argentina (Bonder y Rosenfeld, 2004; Pinkasz y Tiramonti, 2006; Faur, 2008) como al resto de América Latina, mencionaron el crecimiento de la matrícula femenina, su sub-representación en las carreras de ciencia y tecnología (especialmente las ingenierías) y un predominio en las carreras que muestran relación con tradicionales roles femeninos atribuidos en la esfera doméstica (Ovando Crespo, 2007; Graña, 2008). Carreras altamente feminizadas han sido, por ejemplo, la docencia, la enfermería, la obstetricia, asociadas con los roles de cuidado más tradicionales atribuidos a las mujeres, al tiempo que resultaron jerárquicas y salarialmente infravaloradas.

Según un informe de la ONU, difundido el pasado 8 de marzo de 2021, la matrícula femenina en la enseñanza superior se ha triplicado desde la Conferencia de Beijing de 1995. En muchos lugares del mundo las mujeres son más que los varones en Educación Superior, y América Latina es uno de esos espacios. La información proporcionada por la Secretaría de Política Universitaria de Argentina para la fecha arroja guarismos semejantes y en la misma línea van los datos relacionados con nuestra propia universidad. 
Según un informe de la ONU, difundido el pasado 8 de marzo de 2021, la matrícula femenina en la enseñanza superior se ha triplicado desde la Conferencia de Beijing de 1995. En muchos lugares del mundo las mujeres son más que los varones en Educación Superior, y América Latina es uno de esos espacios. La información proporcionada por la Secretaría de Política Universitaria de Argentina para la fecha arroja guarismos semejantes ${ }^{4}$ y en la misma línea van los datos relacionados con nuestra propia universidad 5 .

Las mujeres estudian más, se gradúan más e inclusive más rápido que los varones, sin embargo siguen existiendo asimetrías y desventajas respecto de los accesos a las mejores posiciones laborales y en consecuencia reciben menores salarios. Específicamente a la Educación Superior, son minoría en los espacios de decisión más elevados, (Cargos de rector y vicerrector) En los cargos de docencia e investigación, son mayoría en la base de la pirámide pero a medida que se asciende en el escalafón su número se reduce drásticamente sobre todo en la categoría más elevada.

Ahora bien, si hacemos zoom en la matrícula femenina de grado en la Educación Superior veremos que la distribución no es equitativa en todas las carreras, que hay más mujeres en el área de las Humanidades, las Ciencias Sociales y las Ciencias de la Salud, pero la paridad se invierte en carreras de las ciencias duras, Informática, Tecnología, Matemáticas e Ingenierías. Ciencias Agropecuarias, Ciencias del Suelo. Es decir hablamos de una distribución desigual e inequitativa en sentidos horizontal y vertical (SPU 2019-2020).

Consideramos que el complejo fenómeno de los condicionantes de género debe examinarse en relación con otros ámbitos de la vida social además de la ES. Para comprender las trayectorias educativas sexuadas es necesario ampliar la mirada más allá, al contexto social y

$4 \quad$ En el informe titulado Mujeres en el sistema universitario argentino 2019-2020 difundido por la Secretaría de Políticas Universitarias con motivo de la conmemoración del día de la mujer trabajadora el 8 de marzo se aportaba gran cantidad de información de la que aquí solo se incluye un extracto. Respecto al año académico 2019, las estadísticas dan cuenta de la mayor participación de mujeres en el total de estudiantes, en el total de las/os graduadas/os y también en el total de las/os nuevas/os inscriptas/os que iniciaron carreras durante ese año. En todas ellas, las mujeres representan cifras cercanas al $60 \%$ del total de cada categoría. Este escenario se verifica tanto en los niveles de pregrado y grado como en las carreras de posgrado Es decir, nuestra matrícula universitaria está conformada mayoritariamente por estudiantes mujeres; cada año se inscriben más mujeres que hombres y, además, las mujeres se gradúan más que los hombres, especialmente en los niveles de pregrado y grado. Si bien es notable la presencia destacada de las mujeres en el total del sistema, al realizar un análisis por rama de estudio se observa que en algunos campos disciplinares - especialmente en el de las ciencias aplicadas -su presencia es aún escasa. En contrapartida, se destaca la participación de las mujeres en las ciencias de la salud y las ciencias humanas: las egresadas de las carreras de pregrado y grado representan el $75,2 \%$ y el $76,4 \%$, respectivamente.

En cuanto a los recursos humanos de las universidades nacionales, vale destacar que existe paridad de género entre los cargos docentes y no docentes. Sin embargo, dicha paridad se desajusta cuando se analiza la distribución según cargos y género de autoridades superiores, donde las mujeres representan el $42,8 \%$. Además, se debe prestar especial atención al hecho de que a medida que se incrementa la jerarquía del cargo, disminuye la participación de las mujeres. Tal es así que podemos observar que existen $47 \%$ de mujeres vice decanas, 35\% decanas, 33\% secretarías de Universidad, 31\% vicerrectoras y solo $11 \%$ rectoras/presidentas. P 4 . SPU (2021)

5 Secretaría de investigación. Participación de la mujer en el Sistema de I+D de la UNQ datos relativos a docentes de planta y becarios de la UNQ - Informe 2021 
político. Para entender las autopercepciones, estereotipos y expectativas que emergen en las elecciones de las estudiantes universitarias a distancia es necesario pensar en sus trayectorias educativas previas y cómo éstas contribuyeron a moldear sus disposiciones y las posiciones que aspiraron a ocupar y las que ocupan.

Para entender las autopercepciones, estereotipos y expectativas que emergen en las elecciones de las estudiantes universitarias a distancia es necesario pensar en sus trayectorias educativas previas y cómo éstas contribuyeron a moldear sus disposiciones y las posiciones que aspiraron a ocupar y las que ocupan.

Entenderemos aquí por relaciones de género la organización social de la diferencia sexual. Lo que no significa que el género refleje o instaure las diferencias físicas, naturales y establecidas; sino que más bien es el conocimiento el que establece los significados de las diferencias corporales entre varones y mujeres. La diferencia sexual no es la causa originaria de la cual deriva la organización social, por el contrario ésta diferencia tiene su origen en la organización social variable. El eje está en preguntarse sobre la forma en que se construyen y legitiman jerarquías como las de género. (Scott 2008) La perspectiva así asumida no piensa solamente en las mujeres, sino que propone un enfoque relacional que permita analizar, para superar, las jerarquías establecidas a partir de la construcción binaria de lo femenino y de lo masculino, sin perder de vista las exclusiones que el binarismo ${ }^{6}$ conlleva. Jerarquías que al mismo tiempo producen, conceptualizan y sancionan otras identidades sexuales y genéricas (gays, lesbianas, trans, bisexuales e intersex). (Vasquez y Lajud 2016, Korol 2016)

Consideramos que a partir de estos ejes se debe contemplar las experiencias universitarias virtuales sexuadas, considerando modalidades de estudio y carreras, las identidades particulares y las especificidades culturales de los grupos que acceden a los estudios universitarios.

\footnotetext{
$6 \quad$ Independientemente de que en Argentina desde 2012 fue promulgada la ley 26743 de Identidad de Género, no ha permeado a todas las instituciones de la misma manera. En la Universidad Nacional de Quilmes la resolución 377/19 del Consejo Superior de la Universidad de Quilmes dispuso “Establecer que todas las dependencias académicas, administrativas y de toda índole de la Universidad Nacional de Quilmes deberán reconocer, la identidad de género adoptada o autopercibida de cualquier persona a su solo requerimiento aunque no coincida con su nombre y sexo registrales" con base en el artículo 12 de dicha ley, se establecieron los procedimientos administrativos para dar cumplimiento efectivo.

El sistema de administración académica SIU se ha mantenido incólume, heterosexual y binario en sus registros hasta el 21 de abril de 2021 dia en el que a través del trabajo articulado de varios organismos del Estado, se presentó una propuesta para incorporar la variable de identidad de géneros y diversidad en los diferentes módulos del Sistema de Información Universitaria (SIU) del Consejo Interuniversitario Nacional (CIN), en cumplimiento de la Ley N²6.743 de identidad de género.[...] Fruto de esta tarea, en todo el Ecosistema SIU, como por ejemplo en el módulo de estadísticas de alumnos SIU-Araucano y el de gestión académica SIU-Guaraní, se incorporarán, dentro de los relevamientos de información y de los formularios en los que se deban completar datos personales, diversas categorías que reconocerán las distintas formas en que las personas pueden percibir su género. recuperado online de: https://www.cin. edu.ar/perspectiva-de-generos-y-diversidad-en-los-sistemas-siu/
} 
El modelo pedagógico del Programa Universidad Virtual de Quilmes (UVQ), vigente con modificaciones desde 1989, se caracteriza por su flexibilidad tanto respecto a la organización del recorrido académico (que no prescribe asignaturas correlativas) como de las condiciones de acceso, también por las oportunidades en la organización de tiempos de cursada y estudio según las necesidades del estudiante. El proceso de enseñanza y aprendizaje saca del centro de la escena la coincidencia espacio temporal entre docentes y estudiantes, incorpora la mediación tecnológica a través del aula virtual, promueve mayor autonomía de los aprendizajes, horizontalidad en las interacciones y asincronía.

Nos preguntamos entonces en este trabajo ¿dejar de lado la presencia física implica dejar de considerar en el proceso de aprendizaje el cuerpo sexuado? ¿Siguen operando relaciones asimétricas de poder? Y si es así ¿cómo?

\section{Nos preguntamos entonces en este trabajo ¿dejar de lado la presencia física implica dejar de considerar en el proceso de aprendizaje el cuerpo sexuado? ¿Siguen operando relaciones asimétricas de poder? Y si es así ¿cómo?}

Gran parte de las carreras ofrecidas en modalidad virtual de UVQ son ciclos de complementación de grado, es decir que permiten el acceso de estudiantes con más de quince asignaturas aprobadas de carreras universitarias, tecnicaturas o títulos terciarios afines, lo que lleva a pensar en trayectorias educativas extendidas y estudiantes con edades superiores a los 25 años.

Si bien el logro de la titulación de grado se presenta como un fuerte motivador en el acceso, las experiencias previas portadas no implican conocimientos respecto de Entornos Virtuales de Enseñanza y Aprendizaje (EVEA) lo que resulta un dato importante a la hora de analizar las trayectorias.

En líneas generales podemos afirmar que, en el período estudiado, nuestros y nuestras estudiantes contaban con una edad promedio de 33 años y las mujeres no solo eran más en cantidad, sino que presentaban mejor desempeño en sus notas y exámenes aprobados. Respecto de sus estados civiles el promedio más alto aparecía en el rubro "divorciada", en cambio en los varones eran los "casados" quienes tenían mejores desempeños.

¿Podría interpretarse que operaban los tradicionales roles de cuidado y soporte familiar asignados a las mujeres? ¿Cuál era la distribución 
por sexos entre los estudiantes por carrera? ¿Había carreras con predominio masculino o femenino?

En la observación de la distribución de varones y mujeres en el interior de las carreras los porcentajes mostraron feminización. Dentro del departamento de Economía y Administración, las mujeres constituían el $62 \%$ de la población en la carrera de Contador Público Nacional (CPN); en la Licenciatura en Administración Hotelera (LA) el 55\% y en la Tecnicatura en Ciencias Empresariales el 54\% (TUCE). En la Licenciatura en Comercio Internacional (LCI) los porcentajes alcanzaban el 50\% para cada uno. En el departamento de Ciencias Sociales, las Licenciatura en Educación (LE) y Terapia Ocupacional (TO) poseían porcentajes superiores al $80 \%$ (85 \% y 93\%) y en Licenciatura en Humanidades y Ciencias Sociales (LHyCS), quienes revestían identidades genéricas femeninas eran el $60 \%$. La comparación con informes anteriores mostró una tendencia a la feminización sostenida.

Teniendo en cuenta lo anterior se asumió que la información estadística resulta insuficiente para arriesgar razones o fundamentos detrás de las elecciones que animaron las trayectorias, por tanto para asomarnos a las y los actorxs y a sus decisiones se realizaron en 2017, 34 entrevistas en profundidad a egresados y egresadas. Destacamos que si bien se buscó mantener la proporción entre varones y mujeres por carrera, no se trató de una muestra representativa sino intencional.

Profundizando en los testimonios de varones y mujeres, desde la perspectiva de género el análisis llevó a identificar en los relatos femeninos lo que se entiende por división sexual del trabajo.Tomemos por ejemplo el testimonio de A.v', de la LHyCS, cuando la entrevistadora preguntó por su situación familiar y la cantidad de horas semanales dedicadas a las actividades de estudio y a las hogareñas respondió: "La actividad hogareña en ese momento prácticamente la hacía mi señora, yo no me ocupaba de las actividades hogareñas porque ese tiempo lo dedicaba mucho a trabajar afuera de mi casa y eso permitía tener tiempo para estudiar, por ejemplo en la Universidad Nacional de Quilmes. Digamos que me ayudaba mi señora para así tener tiempo para estudiar". Podríamos pensar en una esposa dedicada al hogar, pero luego al referirse a ella A.v dijo: “Bueno, mi señora que es psicóloga y aparte es magister en salud mental." $Y$ significativamente cuando se le preguntó si ser varón o mujer influyó de alguna manera en su trayectoria la respuesta fue:

"¡Qué pregunta! La verdad que no creo. Yo creo obviamente en la igualdad de la mujer y del hombre y estoy totalmente en contra del machismo patriarcal y toda esa venialidad sobre la mujer que me parece criminal. Pero la verdad que no creo que haya sido así, que el sexo

$7 \quad$ Para preservar la identidad de los entrevistados se usa una la siguiente codificación: la primera letra mayúscula corresponde al nombre y la siguiente v o m implica varón/mujer. 
determine, si bien es una construcción social la elección sexual. En el caso mío ¿me preguntas a mí como hombre? Yo diría que no, que no fue así. Si elegí la carrera en Quilmes fue a partir de una posición ideológica ante la vida y no a partir de ... de sexo, no. Fue una cuestión ideológica y existencial."

En cambio los relatos femeninos discurren por otros carriles, así C.m de la $L E$, luego de decir que no dedicaba tiempo a los quehaceres domésticos porque pagaba a una persona que los hiciera, indica que eligió la modalidad: "Porque los chicos eran chicos. No podría viajar. Realmente me implicaba muchísimo más tiempo y dinero. Entonces no lo podría haber sostenido el ir a cursar en otro lugar."

N.m de la LHyCS, así como otras graduadas de diferentes carreras, indicó:" [..] me quede con un poquito de ganas de seguir estudiando. [...] Pero la verdad es difícil cuando se tienen chicos, se está casado y encima se tiene que trabajar. No se puede cumplir con los horarios de una cursada."

De esta forma reflejó, en cambio S.v de LE su situación familiar "Hubo un momento complicado porque ella quedó embarazada y tuvimos unos altercados con eso, y nos cambiaron mucho los tiempos. Pero pudimos seguir". No sabemos cómo interpretar el término "altercados" con respecto al embarazo, ni cómo podríamos entender que su esposa "quedó" embarazada como algo que solo le hubiese ocurrido a ella. Lo que sí se hace evidente en los relatos femeninos es el peso del trabajo reproductivo como tareas domésticas, burocráticas y cuidado de la familia, además de trabajar fuera de sus casas y estudiar. A.m, egresada de la LE comentó "Le dedicaba poco y nada a las actividades hogareñas. Tengo 45 hs. semanales de clases. Mi marido me ayudaba un montón. Estudiaba de noche. Después de cenar me sentaba a estudiar. El formato que tiene la carrera, los profesores ayudaron a que yo no abandonara".

Varios varones ayudaban con las tareas domésticas, sin embargo sus relatos respecto de la organización del tiempo discurrieron entre el dedicado al estudio (o falta de tiempo para ello) y a las horas destinadas a sus actividades laborales. El eje central estuvo puesto en su participación en el espacio público ya sea con tareas de carácter remunerado, participación social o cultural. G v de la LE explicó “Era que ya tenía trabajo, entonces ya estaba establecido laboralmente, después también mi familia porque me ayudaban en el tiempo que necesitaba para terminar la carrera pese a que yo ya tenía terminada otra carrera y las posibilidades de poder ampliar el campo laboral después."

Cuando se atendió a las interrupciones en la carrera, aquellas estudiantes que las tuvieron hicieron referencia a enfermedades de los hijos, familiares convivientes o picos de estrés, los varones a cuestiones laborales o bien a exceso de actividades públicas como evidencia el siguiente testimonio: 
"Yo hago mucha actividad comunitaria y ambiental en (mi ciudad). Me gusta compartir el tiempo existencial con el tiempo en la sociedad que vivo, en la medida que puedo. La que me banca es mi compañera, mi pareja, por eso puedo. Y bueno sacando esos casos que a veces interrumpía o porque yo rendí una vez mal, después no hubo interrupciones. O sea que fue por trabajo, por salud y después retomaba". (A.v LHyCS)

También H.v, casado y con dos hijos, refirió a que su trayectoria fue bastante constante, tuvo "interrupciones laborales pero la mayoría de la carrera fluyó." (El resaltado es nuestro). Por el contrario a la pregunta sobre sus condiciones familiares cuando estaba estudiando, una graduada de TO señaló que "convivía con su esposo y sus dos hijos y dedicaba aproximadamente entre 20 y 25 horas semanales a las actividades hogareñas". Sobre su trabajo remunerado dijo que trabajaba "entre 20 y 30 horas semanales. Los horarios eran fijos, de manera que no tenía flexibilidad para acomodar sus tiempos respecto de los estudios". Sobre el apoyo recibido para desarrollar su carrera refirió a su familia y agregó "con quienes tuve que cambiar bastante seguido los roles para llevar adelante las actividades hogareñas", lo que nos permite advertir que consideraba las tareas hogareñas como propias, y si nos quedaban dudas agrega más adelante que: "la modalidad fue una ventaja por brindarle la posibilidad de estudiar sin dejar de trabajar ni desatender a su familia". Finalmente a la pregunta: -“ ¿Pensás que ser mujer/varón tuvo algo que ver con el desarrollo de tu trayectoria o con la elección de tu carrera?" Responde con un rotundo no para ambos aspectos.

Otra egresada de la misma carrera indicó que durante el proceso de estudio "convivía con su esposo, dos de sus cuatro hijos y dos tías y que dedicaba aproximadamente entre 40 y 50 horas semanales a las actividades del hogar, por contar con una familia numerosa." A la pregunta sobre los motivos de elección de la modalidad de cursado respondió que "eligió la modalidad virtual por tener una familia numerosa y trabajo, lo que le imposibilitaba continuar sus estudios de manera presencial." Y ante la pregunta sobre si su condición de mujer tuvo relación con la elección de la carrera o la trayectoria respondió que no.

En palabras de M.m, de la carrera de Contador Público Nacional (CPN), respecto de las dificultades que enfrentó para obtener su título "Además de los problemas de salud por los que pasó mi marido, la mayor dificultad fue trabajar, mantener la casa y la familia y estudiar, todo al mismo tiempo. Se complicó mucho, especialmente teniendo tres hijas adolescentes".

Todo lo anterior nos lleva a incorporar el concepto de triple jornada para explicar las trayectorias femeninas. La mujer trabajadora no solo desempeña sus actividades un lugar determinado sea este una fábri- 
ca, una empresa o una escuela, sino que luego continúa desarrollando un trabajo familiar no remunerado y también un trabajo afectivo de producción de vínculos y redes de cuidado (Gago, V 2019). De este modo en el espacio doméstico la mujer reproduce las condiciones de posibilidad de libertad del varón y reduce las suyas, tal como se hizo presente en el testimonio de A.v (LHyCS).

\section{Todo lo anterior nos lleva a incorporar el concepto de triple jornada para explicar las trayectorias femeninas. La mujer trabajadora no solo desempeña sus actividades un lugar de- terminado sea este una fábrica, una empresa o una escuela, sino que luego continúa desarrollando un trabajo familiar no remunerado y también un trabajo afectivo de producción de vínculos y redes de cuidado (Gago, V 2019).}

Las mujeres entrevistadas dividían su tiempo para trabajar fuera y dentro de la unidad doméstica algo que, aún hoy, no parecería suceder con los varones. Y a la vez la triple jornada femenina impide, o dificulta, la realización de otras actividades como estudios, o intervenciones políticas o socioculturales. Al invisibilizarse una parte de las tareas cumplidas por ellas se produce un proceso de naturalización y esencialización de lo que se entiende por atributos femeninos en el imaginario colectivo, como si existieran por naturaleza determinadas tareas para las que las mujeres son especialmente aptas, como aquellas que constituyen una prolongación de la asistencia realizada en el ámbito doméstico o comunitario y se las excluye de otras que tienen que ver con la toma de decisiones, la planificación, y la organización de tareas profesionales (Bonaccorsi, 2007,P: 103).

Del análisis de la totalidad de las entrevistas se desprende algo que las feministas han identificado hace tiempo: la condición de mujer y la toma de conciencia y defensa de sus derechos de igualdad de oportunidades no van de la mano. Ni se puede pensar en representaciones de sí que escapen a la atribución de una esencia femenina provista de características específicas:

[...] vos sabes que, no conozco las estadísticas pero que por ahí las mujeres tienen mayor ímpetu en que aunque son grandes, seguir estudiando y los hombres no. Y creo que las mujeres son más guerreras que los hombres, por naturaleza. Entonces eso las impulsa a seguir, a seguir, y aunque son mamá y son grandes, a desarrollarse ellas mismas. Y los hombres si lo logran o lo hacen es porque tal vez las mujeres los impulsan. Eso sí lo veo. (G.m LE) 
dirección por ejemplo S.m de la LE a la pregunta ¿vos pensás que ser de mujer, tuvo que ver con el desarrollo fluido en la carrera o la elección de la misma?"Sí, creo que es un factor. Una trabaja y tiene familia, se dedica a los hijos y creo que para el varón tal vez es más fácil realizar una carrera aunque sea dentro de la docencia". A la misma pregunta L.v de la LE respondió: "No lo analicé nunca y tampoco me encontré en alguna situación en la que así lo pareciera. Sí en el estudio anterior que tuve, que estudié magisterio, éramos 1 (varón) cada 50 (mujeres), me sentí que me facilitaban las cosas solo por ser varón. Pero en la Universidad de Quilmes, ahí no."

De la totalidad de los varones entrevistados, a excepción de L.v, ninguno manifestó que las relaciones de género y las tensiones entre las categorías implicadas hubiesen tenido alguna repercusión en sus trayectorias, el análisis no mostró lo mismo, los casos aquí incluidos funcionan como ilustraciones breves de la totalidad que discurrió en la misma línea. Entre las mujeres, se pudo identificar que el doble y triple trabajo, sin poner en cuestión los roles domésticos tradicionales y el cuidado de los hijos y otros familiares, siempre ha sido compatibilizado con los estudios.

No sucedió lo mismo con los varones, en sus casos los condicionantes procedieron de las situaciones de falta de flexibilidad laboral o el tiempo dedicado al mismo, la familia funcionó como apoyo o complemento. En la mayor parte de los casos las mujeres plantearon una situación de igualdad con sus compañeros que no podemos más que cuestionar, y en los pocos casos en los que manifestaron dificultades enfrentadas por ser mujeres, para conseguir trabajo o para desarrollar los estudios, no pusieron en cuestión que el cuidado de los hijos fuera una tarea que les correspondía primordialmente a ellas. La vida cotidiana parece ser bastante más refractaria a la igualdad de lo que podría pensarse desde el sentido común de la sociedad argentina en el siglo XXI. Y la Universidad se encuentra lejos de reconocer las condiciones sexuadas de las trayectorias.

\section{La vida cotidiana parece ser bastante más refractaria a la igualdad de lo que podría pensarse desde el sentido común de la sociedad argentina en el siglo XXI. Y la Universidad se encuentra lejos de reconocer las condiciones sexuadas de las trayectorias.}

Pensamos que es necesario interpelar estas experiencias tanto desde los aportes de la perspectiva de género, como de los estudios queer y de las pedagogías feministas en la idea de politizar aquello que ha sido tradicionalmente reducido a la esfera de lo privado y colocarlo en el foco para analizar la discriminación o las diferencias en 
las oportunidades y para traerlo a las reflexiones que la universidad hace respecto a sus estudiantes y egresados. Resulta oportuna la propuesta de Luz Maceira Ochoa quien desde la pedagogía feminista propone identificar "los recursos materiales, subjetivos y simbólicos que las educandas requieren para transgredir las normas y esquemas que las oprimen, para descautivarse y construir y afirmar su mismidad, por tanto, su punto nodal es la persona, la sujeta, y como proceso formativo se interesa tanto por los saberes como por los poderes de esa sujeta" (Morgade 2016, P.251)

\section{RECAPITULACIÓN FINAL}

Pensamos que las entrevistas analizadas avalan la idea de que la presencia femenina, sus elecciones y sus trayectorias en la matrícula universitaria (aunque la investigación focaliza en entornos virtuales creemos que pueden iluminar decisiones de otras modalidades) deben ser entendidas en el nivel de la política en sentido amplio, en cuanto a relaciones de poder y contextualizada históricamente, tal como refiere Scott (2008). Podemos relacionar esta presencia con los cambios en las expectativas femeninas que se sucedieron a partir de la década de 1960, sin considerar que los avances han sido absolutos. Las mujeres que optaron y optan por acceder a un trabajo y/o a la universidad están mostrando mayores márgenes de autonomía, en distintos niveles de su existencia y también dan cuenta de cambios ocurridos en las autopercepciones de sus derechos y en sus expectativas de ascenso social.

Sin embargo las entrevistas nos muestran que estos avances van a acompañados de fuertes tensiones por la vigencia que aún tienen los roles femeninos y masculinos tradicionales, sobre todo respecto de las obligaciones en el interior de las parejas o en lo referente a la maternidad. Estos estereotipos no son cuestionados por estudiantes adultos/ as que presentan trayectorias educativas extensas donde estos desequilibrios y asimetrías no parecen haber sido puestos en cuestión, sino más bien todo lo contrario, tal como señalan autoras y autores que analizan la situación escolar (Morgade 2016, Vasquez Lajud 2016, Korol 2016, Guerrero 2005, Maceira Ochoa 2008) o el ámbito Universitario (Pinkasz Tiramonti 2006, Ovando Crespo 2007, Graña 2008, Bonder y Rosenfeld 2004, Faur 2008, solo para nombrar algunos) resultan reforzados.

Las diferencias en los accesos, los estudios seleccionados y los espacios ocupados, muestran que la presencia masiva de mujeres no implica el fin de las desigualdades de género y evidencian una estructura patriarcal que se mantiene fuerte, aunque, pensamos, no indemne. En el caso particular de la UVQ, las entrevistas permiten identificar declaraciones de igualdad tanto en varones como en mujeres conviviendo 
con ideales de género tradicionales operando en los y las estudiantes, por lo que consideramos que las desigualdades residen no solo en la Universidad sino también más allá de sus límites y, en consecuencia, debe llevarse la mirada al proceso de construcción identitaria genérica que se produce en el flujo ininterrumpido de los aprendizajes y que se inicia muy precozmente en el seno familiar, se continúa luego en la escuela y en el resto de la vida social.

Pero también más recientemente, con la presencia de los feminismos en las Universidades donde surgen nuevos reclamos y se institucionalizan las iniciativas relacionadas con la defensa de los derechos de las mujeres y disidencias sexuales, nos planteamos la necesidad imperiosa de que la perspectiva feminista atraviese la malla curricular universitaria, de lo contrario continuaremos permitiendo la existencia de un relato esquizoide respecto de una experiencia igualitaria donde en realidad hay desigualdad y con bellas declaraciones de equidad impartimos conocimientos científicos desde una perspectiva universal, pretendidamente neutra y que es en realidad androcéntrica y heterosexuada. De lo contrario no contribuiremos a entregar herramientas que permitan a las sujetas descautivarse, ni promoveremos revisión de masculinidades normativas o arquetípicas (Jones, Blanco 2020), ni aperturas a las disidencias.

Hasta donde hemos llegado en nuestra investigación, los entornos virtuales no presentan grandes diferencias con otras ofertas de Educación Superior ni en cuanto a presencia, performance ni graduación femeninas. Y contrariamente a lo que podría pensarse en la virtualidad las corporalidades también se hacen presentes y el binarismo que se mantiene.

No perdemos de vista que se trata del análisis de un caso particular de ES a nivel de grado en EVEA, sin embargo hemos podido establecer relaciones con estudios similares en ámbitos universitarios nacionales e internacionales, lo que permite pensar en avanzar un poco más allá del caso particular e iluminar en cierto modo decisiones y perspectivas que atañen a varones y mujeres cuando deciden encarar estudios superiores. Y enfocar las tensiones que se generan entre estas categorías, los márgenes de autonomía, los espacios de agencia y de reproducción que conviven con las identidades y comportamientos esperados o disponibles y como son apropiados por las y los actores. 


\section{BIBLIOGRAFIA}

Bracchi, C; Gabbai,M.I (2013). Subjetividades juveniles y trayectorias educativas: tensiones y desafíos para la escuela secundaria en clave de derecho, en Kaplan, C (Comp) Culturas estudiantiles. Sociologá de los vínculos en la escuela. CABA: Miño y Dávila

Bonaccorsi, N. (2007). Doble jornada laboral, en Gamba, S.(coord.)(2007). Diccionario de estudios de género y feminismos. Buenos Aires: Biblos.

Bonder, G; Rosenfeld, M. (2004). Equidad de género en Argentina. Datos, problemáticas y orientaciones para la acción. Programa de las Naciones Unidas para el Desarrollo (PNUD) Argentina. Recuperado de(21/04/2016): http:// www.dhl.hegoa.ehu.es/recursos/121

Faur, L (2008). Desafíos para la igualdad de género en la Argentina. Buenos Aires, Programa Nacionas Unidas para el Desarrollo. PNUD

Gago, Verónica (2019). La potencia feminista o el deseo de cambiarlo todo. CABA, Tinta Limón.

Graña, F. (2008). El asalto de las mujeres a las carreras universitarias "masculinas": cambio y continuidad en la discriminación de género. PRAXIS Educativa, 12(12), 77-86. Recuperado de (20/03/2017): http://www.redalyc.org/articulo. oa?id=153112902008

Haraway, D (1995). Ciencia, ciborgs y mujeres. La reinvención de la naturaleza. Madrid, Cátedra.

Jones, D; Blanco Rafael (2021). Varones atravesados por los feminismos. Deconstrucción, distancia y reforzamiento del género en: en Fabbri Luciano (Comp) La masculinidad incomodada

Korol, C (Comp) (2016). Feminismos populares: pedagogías y políticas. CABA, Chirimbote.

Lorenzo, M. F. (2016). Han recorrido un largo camino muchachas. La participación femenina en la Universidad de Buenos Aires entre 1940 y 1965. En: Que sepa coser, que sepa bordar, que sepa abrir la puerta para ir a la universidad. Buenos Aires: EUDEBA.

Martínez Ramos, L.(et al.) (2007). Representación por género en Educación Superior. Informe de investigación. Proyecto de participación y representación por género en Educación Superior. Universidad de Puerto Rico, Río Piedras, 3-148. Recuperado de: http://www2.pr.gov/agencias/cepr/inicio/ 
Morgade, G (2016). Cuerpos sexuados en la escuela secundaria: política y políticas en una escuela que deviene otra; en Brener, G; Galli, G (Comp) Inclusión y calidad como políticas educativas de Estado: o el mérito como opción única del mercado.CABA: Asociación Educacionista Argentina Editorial Stella.

Ovando Crespo, C. (2007). Género y educación superior. Mujeres en la docencia y la administración en las instituciones deeducación superior. En: Escenarios mundiales de la educación superior. Análisis global y estudios de casos. Recuperado de http://bibliotecavirtual.clacso.org.ar/ar/libros/campus/segrera/050Crespo.pdf

Pinkasz, D. y Tiramonti, G. (2006). Las oportunidades educativas de las mujeres en la modernización de los 90 en Argentina. Equidad de género, 51. Recuperado de (30/05/2016): ww.oei.es/reformaseducativas/equidad_genero_reformas_educativas.pdf

Scott Wallach, J. (et al.)(2008).Género e historia. México: Fondo de Cultura Económica.

Vasquez, E; Lajud C (2016) Identidades y diversidades de género en la Escuela. Desafíos en pos de la igualdad, en: Kaplan, C (Comp) El género es más que una palabra. Educar sin etiquetas. CABA: Miño y Davila. 